\title{
Conditions Facilitating the Aversion of Unpopular Norms: An Agent-Based Simulation Study
}

\author{
Zoofishan Zareen \\ Bahria University \\ Islamabad, Pakistan
}

\author{
Muzna Zafar \\ Bahria University \\ Islamabad, Pakistan
}

\author{
Kashif Zia \\ Bahria University \\ Islamabad, Pakistan
}

\begin{abstract}
People mostly facilitate and manage their social lives adhering to the prevalent norms. There are some norms which are unpopular, yet people adhere to them. Ironically, people at individual level do not agree to these norms, but, they still follow and even facilitate them. Irrespective of the social and psychological reasons behind their persistence, sometimes, for societal good, it is necessary to oppose and possibly avert the unpopular norms. In this paper, we model theorydriven computational specifications of Emperor's Dilemma into an agent-based simulation, to understand the the conditions that result in emergence of unpopular norms. The reciprocal nature of persistence and aversion of norms, thus, is utilized to define situations under which these norms can be changed and averted. Simulation is performed under many interesting "what-if" questions. The simulation results reveal that under high density conditions of agent population with a high percentage of norm aversion activists, the aversion of unpopular norms can be achieved.
\end{abstract}

Keywords-Agent-based Modeling and Simulation; Emperor's Dilemma; Complex Adaptive Systems.

\section{INTRODUCTION}

Norms are regular principles of conduct that organize our communications with others. Norms plays an important role in the development of the individual behavior and the social order [1]. Since, norms affect individual and group behavior, emergence of behavior at societal level reform the norms. Hence, norms and their manifestation on the society are related to each other in a reciprocal manner, being influenced and influencing each other [2].

Intuitively, norms are supposed to be the conventions and rules which prevail in a society due to their goodness at the societal level. However, the factors influencing the emergence of such a collective thought are not homogeneous and fair in nature. Sometimes, the human factors of fear, greed, honor, influence, and belief etc., collectively manifest into a norm which is not really popular, but still prevails. While, an unpopular norm emerges, majority of the people at individual level do not agree with that, however, due to social pressure they have no option but to adhere to it. Surprisingly, they even become advocate of the norm due to potential embarrassment as a consequence of revealing their private thought to a group of people which they think, think otherwise. Some interesting examples quoted in the literature are foot binding [3], female genital mutilation [4], Johannes town massacre [5] and acceptability of corruption [6].
To reason about emergence of norms in a society, two predominant approaches are: economic and social approach The economy-based approach resides on the basics of costbenefit analysis, where each individual or group is required to be "somewhat" rational. For many, this cannot be true for a human society. Hence, the social approach focus on societal factors which effect the decision-making of individuals and groups, such as preferences, networking and interactions, and cooperation and externalities [7]. Naturally, the social approach is better to reason with unpopular norms.

A general description of using Agent-Based Modeling $(\mathrm{ABM})$ as a bottom-up approach for modeling social interactions is presented in [8]. A more generic study with focus on cultural differentiation based on social factors of homophily, influence and network structure is presented in [9]. Similar studies exists throughout the research literature [10], [11], [12], [13], [14], [15].

The seminal work by Damon Centola, Robb Willer and Michael Macy [16] explains the social factors influencing the emergence of an unpopular norm. In this work, they have proposed a computational model of self-enforcing norms, stating the conditions necessary to enforce an unpopular norm. Additional work also exists modeling the compliance and/or enforcement of a an unpopular norm, covered in next section.

In this work, we argue that for societal good, it is necessary to oppose and possibly avert the unpopular norms. Hence, we attempted to realize the conditions that result in emergence of unpopular norms and define situations under which these norms can be changed and averted. To achieve it, we used and extended the social interaction model proposed by Centola, et.al. [16].

\section{RELATED WORK}

There are two cognitive aspects that describe the human reaction to a situation related to norms. First is the compliance; the person confronted with such a situation may decide to comply to the norm or not. The second is the enforcement; the person confronted with such a situation may decide to enforce the norm or not. Related work exists in both directions. Additionally, the methodologies to do research on the topic (and many other related social phenomena) can broadly be categorized into four types: (i) purely theoretical, (ii) experimental (performing experiments on human subjects), (iii) mathematical and (iv) model-based (with agent-based models being most popular). 
Irrespective of whether the norms are popular or not, Helbing, et. al. [17] presented an excellent description of conditions for the emergence of shared norms in populations with incompatible preferences. They have presented an agentbased model and simulated interesting scenarios to reach to following conclusions. First, effects of punishments on emergence of norms by defining behavior-based and preferencebased punishment. Due to punishment there is probability of emergence of norms that is sometimes called unpopular norms. Individuals publicly follow the norms due to fear of punishment but privately oppose these norms. Second, is externalities needed for norm emergence. Externalities help in cooperation. Third, norms emerge due to social networks where everyone interacts. Agents in social networks communicate with each other and try to convince to comply or deviate from current norm. Fourth, path-dependent is important feature of norms. The paper concludes that the agents are influenced by neighbors and they set their preferences according to their neighbors preferences and neighbors current behavior to set a shared norms in a society.

In [18], authors argue that dependency and ease of access of information in the current technological age have given birth to propagation of false beliefs, named by them as "Infostorms". They blame three factors responsible for such untrue storms: informational cascades, bystander effect / pluralistic ignorance and group polarization. This paper gives a theoretical understanding of phenomena assisted by real-life examples. The authors conclude that technological systems developed with epistemic approaches with emphasis on truth tracking can prove to be beneficial to avert the infostorms.

Informational cascades are related with social interactions. These connections can be with ones own social network or some random network. Studying the evolution and emergence of norms influenced by such interactions is an interesting domain. For this purpose, the authors in [19] defined a weighted selection algorithm that determines the probability of a person to meet a stranger on the basis of individuals path distance. Using this algorithm, the paper elaborates four cases. In the first case, the agents take rational decision to opt for a norm based on highest utility. Whereas, in second case the agents use Markov decision process to select a norm by assigning weights. Third and fourth cases focus on examining the effect of social interaction on evolution of norms as it spreads throughout the masses. The simulation results showed that in first case people converge to a single norm but the second case takes lesser time in convergence of the norm. Defining the norm as an n-bit sequence indicated that increasing the random interaction had some adverse effects.

A logic-based approach to pluralistic ignorance is proposed in [20], [21]. Being one of the main reason of propagation of unpopular norms, it is important to define the pluralistic ignorance. It can be defined as a situation where "no one believes, but everyone believes that everyone else believes." The logic behind diffusion of unpopular norms is derived from theoretical understanding and intuition. However, the authors argue that the pluralistic ignorance is a fragile phenomenon such that a simple act of public announcement can suspend it. They further present the conditions of dissolution of pluralistic ignorance by stating that either all agents need to announce or an information from a trusted sources would help.
Beyond compliance, the enforcement of an unpopular norm is also evidenced. It has been observed that people enforce unpopular norm to which they privately disapprove. This paper [22] is based on discovering the reason of false enforcement. The authors are of the opinion that people enforce norms to create an illusion of sincerity rather than conviction. The study has been tested in two experiments of wine tasting and text evaluation. Both experiments reveal that the people who enforced the norm, against their actual belief, under social pressure criticized the deviants of the norm. These outcomes indicate how social pressure can lead to false enforcement of an unpopular norm.

On aversion of unpopular norms, the literature available is quite thin. But, there is a need to work in this direction as it is evidenced [23] that people often are not gratified with the norms that already persist in the society and they want to change it or terminate it. The authors relate the mechanism of change with the reasons of why people obey unpopular norms. First is the lack of accurate information about others' behaviors. Second is the herding effect (do as others do). And the third, is the panic punishment. In this paper researchers discussed that having accurate information through communication can avert the unpopular norms and can counter the first two reasons. Third reason is countered through time interval. Lower the time interval between discussion and outcome, the lower the probability of uncertainty leading to more chances to change unpopular norm. However, the study is experimental without any formal model.

We, in this paper, propose a model of aversion of unpopular norm. It is an agent-based model, facilitating the analysis of interesting scenarios in a systematic manner. The model's motivation comes from [16], in which, an agent-based computational model of Emperor's Dilemma is presented. The model resolves the conflict between compliance and enforcement of a unpopular norm, supported by few true believers and privately disapproved by a majority of disbelievers. The authors quantify the influence of networking and population distribution onto the diffusion of unpopular norms or otherwise. In our model presented in the next section, followed by Centola's original specifications, we focus on possibility of aversion of unpopular norms, introducing the reciprocal of behavior of true believers enforcing the unpopular norm unconditionally.

\section{MODELS}

\section{A. Agent-Based Computational Model of Emperor's Dilemma} [16]

In [16], authors state the Emperor's Dilemma as:

"Hans Christian Andersen ... tells the story of three rogues who sell a foolish monarch a nonexistent robe that they claim cannot be seen by those who are "unfit for office" or "incorrigibly stupid." Fear of exposure leads the emperor, and in turn, each of the citizens, to express admiration for the new clothes, which then reinforces the illusion of widespread support for the norm. The spell is broken when a child, innocent of the norm, laughs at the naked old man."

The agent-based computational model of Emperor's Dilemma, proposed in [16] formalizes the phenomena of spreading of unpopular norms through equations of compliance 


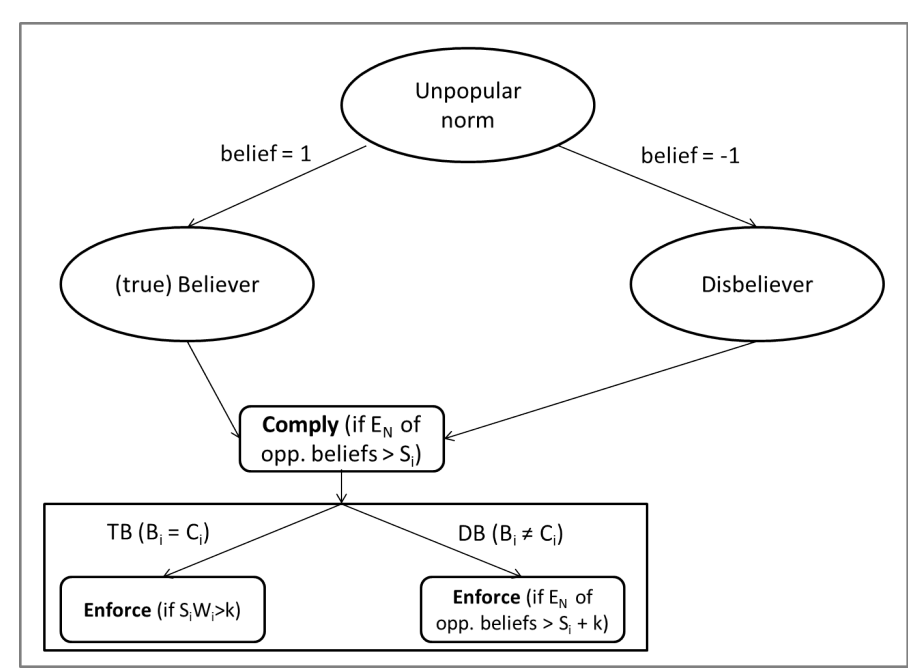

Fig. 1: Flow Diagram of the model based on Emperor's Dilemma [16].

and enforcement. They model two behaviors for the agent; compliance, when agents comply to the norm; and enforcement, when agents, in addition to compliance, also enforce the norm in their region of influence. The factor of compliance transforms an agent either to comply with the norm or choose to deviate from it based on its personal belief, strength of the belief and the neighborhood dynamics. Once complying, an agent may choose to enforce the norm as well.

The model is based on two type of agents; true believers (TB) and disbelievers (DB). Believers are those agents who truly believe in the sanctity of the unpopular norm, while disbelievers are those agents who disbelieve (at least privately) in the sanctity of the the unpopular norm. Hence the belief of an agent on the truthfulness of an unpopular norm corresponds to its type; 1 for $\mathrm{TB}$, and -1 for $\mathrm{DB}$. The initial value of compliance of the norm is also set to 1 for $\mathrm{TB}$, and -1 for DB. The strength of the belief corresponds to how strongly an agent believes what it believes; hence, the value is equal to 1 for $\mathrm{TB}$ and a low random fraction for $\mathrm{DB}$.

In discrete time simulation, each agent $i$ in the simulation space, performs the following actions:

- Interact with the neighbors and calculate the value of Enforcement Need $\left(W_{i}\right)$ as:

$$
W i=\frac{1-\left(\frac{B i}{N i}\right) \sum_{j=1}^{N i} C j}{2}
$$

where $B_{i}$ is agent's belief, $N_{i}$ is neighbors count and $\sum_{j=1}^{N i} C j$ represents the neighbors count whose compliance is not equal to i's belief.

- Comply with the norm if the value calculated below is equal to 1 :

$$
C i=\left\{\begin{array}{cc}
-B i \text { if } & \frac{-B i}{N i} \sum_{j=1}^{N i} E j>S i \\
B i & \text { otherwise }
\end{array}\right.
$$

i.e. compliance $\left(C_{i}\right)$ is set to opposite of the belief, if strength of enforcement of opposite belief by the neighbors is greater than i's own strength; otherwise the $C_{i}$ remains equivalent to agent's belief.

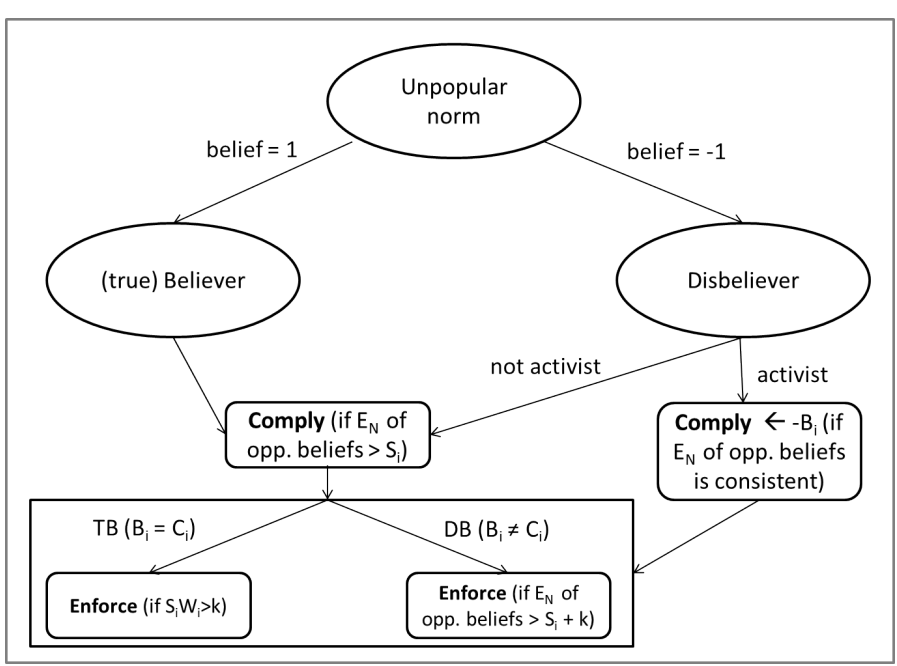

Fig. 2: Flow Diagram of the Proposed Model.

- Enforce the norm; whether it is true enforcement or false enforcement:

$$
E i=\left\{\begin{array}{ccc}
-B i \text { if } & \left(\frac{-B i}{N i} \sum_{j=1}^{N i} E j>(S i+k)\right) \bigwedge(B i \neq C i) \\
+B i & \text { if }(S i W i>k) \bigwedge(B i=C i) \\
0 & \text { otherwise }
\end{array}\right.
$$

i.e. enforcement $\left(E_{i}\right)$ is set to opposite of the belief, if strength of enforcement of opposite belief by the neighbors is greater than i's own strength plus a factor $k$ and current belief is not equal to compliance; otherwise if belief is equal to compliance, and and product of $S_{i}$ and $W_{i}$ is greater than $k$, the $E_{i}$ remains equivalent to agent's belief.

Figure. 1 depicts the transition diagram of this model. An agent whether TB or DB complies with the norm if the enforcements of agents with opposite belief in the neighborhood exceeds its strength. Since, the enforcement values range from -1 to 1 , and we take an average (see equation (2)), for a TB, it can never be more than its strength $(=1)$. Hence, a TB would never be affected by the influence of the neighborhood even when all the neighbors are DBs and are not complying. For a $\mathrm{DB}$, as a result of compliance, the value $C_{i}$ changes to negation of its belief $(=1)$.

All agents who are complying would enforce the norm. For a TB, the value $E_{i}$ would always be equal to $B_{i}$, i.e. 1 , if the value of $W_{i}$ is greater than $k$ (i.e. in the neighborhood, there are sufficient non-complying DBs). The constant $k$ determines the sensitivity of a TB for a need of assertion of norm. A TB would always be applying true enforcement. For a $\mathrm{DB}$, the value $E_{i}$ is equal to opposite of its belief $(=1)$, if there is sufficient enforcement pressure from the surrounding. A TB would always be applying false enforcement.

\section{B. The Proposed Model Extension}

As it is evidenced in the model presented above that a TB is not a normal agent; i.e., it would never be affected by whats happening in the surrounding. Our model is based on reciprocity of this behavior. It means that a minority of DBs are assumed to be more enthusiastic about averting the unpopular 


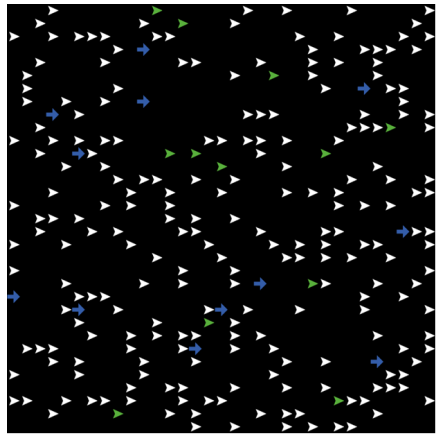

(a)

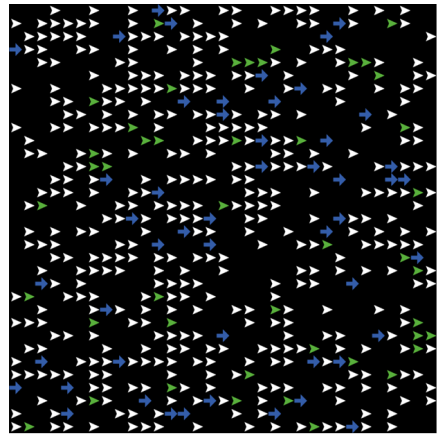

(b)

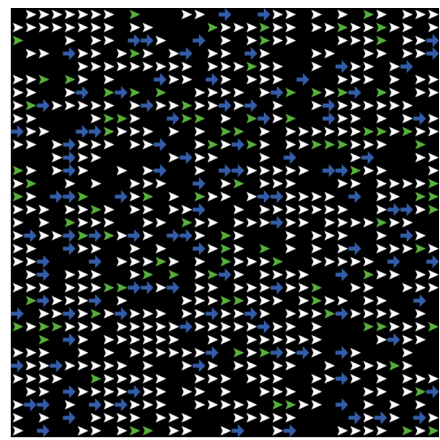

(c)

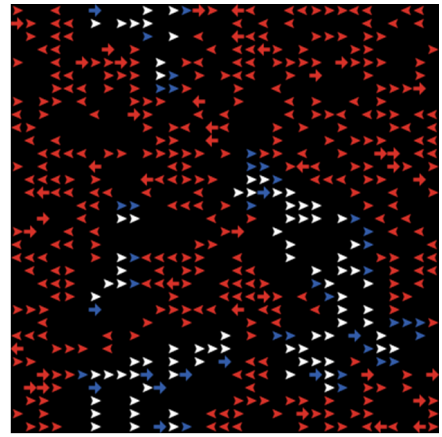

(d)

Fig. 3: Simulation Setup of 3 of 27 cases: agents in white are disbelievers, agents in blue are (true) believers, and agents in green are activists, in a (a) sparse population (250 agents) with TBPerc $=5 \%$ and ACTPerc $=5 \%$, (b) medium dense population (500 agents) with TBPerc $=10 \%$ and ACTPerc $=10 \%$, and (c) dense population (750 agents) with TBPerc $=15 \%$ and ACTPerc $=15 \%$. The ACTPerc is a percentage out of disbelievers. The orientations of all agents are towards "right" initially. (d) Agents' state after end of the simulation of case 14: all agents in red are applying enforcements; even some true believers are applying enforcement to avert the norm.

norm. We name such an agent as an activist (ACT). Like a $\mathrm{TB}$, an activist would never be affected by whats happening in the surrounding. Like TBs, they would be ambitious about fulfilling their role which is acting to avert the norm. The role with be triggered by presence of TBs in the surrounding, particularly who are enforcing.

A transition diagram of the extended model is shown in Figure. 2. An activist would change its belief from -1 to 1 after being encountered by enforcements of norms from the neighborhood. This is achieved by progressive increment of the value of $S_{i}$ by a constant $k$. If this value reaches to 1 or greater, the belief of the agent is changed from -1 to 1 , which means that now the agent believes in aversion of the unpopular norm and acts to avert it. The exact formulation is given in equation (4).

$$
C i=\left\{\begin{array}{cl}
-B i & \text { if } S i>1 \\
B i & \text { otherwise }
\end{array}\right.
$$

Now an activist acts like a true believer and enforces the norm unconditionally (because the value of $S_{i}$ is sufficiently hight already). Since this enforcement will be in the opposite direction, it would be aversion of unpopular norm. Even the TBs are susceptible to be enforced as we will see in the analysis section.

\section{Simulation and Results Discussion}

\section{A. Simulation Setup}

The simulation is performed in Netlogo [24], a popular agent-based simulation tool with grid space support. Netlogo provides an automatic mechanism of applying a function on a set of agents in a random order in successive iterations. Hence a fairness between agents is ensured even though they use the current state of required agents (e.g. the neighborhood of an agent) while making a decision. The agents reside on cells of a spatial grid. We have used Moore's neighborhood to represent the surrounding of an agent which has been a popular strategy in many cell-based spatial configurations [25].
A specific simulation setting is represented as a simulation case, which corresponds to density of the population of agents, percentage of TBs in population TBPerc, and percentage of ACTs in population ACTPerc. For visual assistance, we represent the compliance of unpopular norm with a directional clue. Hence, the agents averting the unpopular norm have a completely opposite direction. The agent type and state is also visually differentiated. This is further explained in Figure. 3. A complete list of simulation cases is given in Table. I. Each simulation case was run for 10 times and the results were averaged.

\section{B. Simulation Results}

The simulation results are analyzed based on four quantities:

- DBAvertPerc: The percentage of disbelievers who ended up averting the unpopular norm.

- DBComplPerc: The percentage of disbelievers who ended up complying the unpopular norm.

- TBAvertPerc: The percentage of true believers who ended up averting the unpopular norm.

- TBComplPerc: The percentage of true believers who ended up complying the unpopular norm.

The Table. I shows the 27 simulation cases and the corresponding values for above measures. Qualitatively the norms is considered as been averted if majority of the population starts refuting it (acting against it). The result verify a trend that can be stated as:

- The percentage of agents averting the unpopular norm increases with increase in population.

- The percentage of agents averting the unpopular norm increases with increase in ACTPerc.

- The percentage of agents averting the unpopular norm increases with increase in relative positive difference between ACTPerc and TBPerc. 
TABLE I: Complete list of all 27 simulation cases.

\begin{tabular}{|c|c|c|c|c|c|c|c|}
\hline Case No. & Population & TBPerc & ACTPerc & DBAvertPerc & DBComplPerc & TBAvertPerc & TBComplPerc \\
\hline 1 & 250 & 5 & 5 & 11.39 & 88.61 & 8.33 & 91.67 \\
\hline 2 & 250 & 5 & 10 & 24.05 & 75.95 & 16.67 & 83.33 \\
\hline 3 & 250 & 5 & 15 & 32.49 & 67.51 & 16.67 & 83.33 \\
\hline 4 & 250 & 10 & 5 & 9.33 & 90.67 & 8 & 92 \\
\hline 5 & 250 & 10 & 10 & 21.33 & 78.67 & 12 & 88 \\
\hline 6 & 250 & 10 & 15 & 28.89 & 71.11 & 24 & 76 \\
\hline 7 & 250 & 15 & 5 & 11.79 & 88.21 & 5.41 & 94.59 \\
\hline 8 & 250 & 15 & 10 & 20.28 & 79.72 & 13.51 & 86.49 \\
\hline 9 & 250 & 15 & 15 & 33.96 & 66.04 & 29.73 & 70.27 \\
\hline 10 & 500 & 5 & 5 & 17.47 & 82.53 & 8 & 92 \\
\hline 11 & 500 & 5 & 10 & 31.79 & 68.21 & 28 & 72 \\
\hline 12 & 500 & 5 & 15 & 48 & 52 & 32 & 68 \\
\hline 13 & 500 & 10 & 5 & 14.89 & 85.11 & 4 & 96 \\
\hline 14 & 500 & 10 & 10 & 31.78 & 68.22 & 24 & 76 \\
\hline 15 & 500 & 10 & 15 & 48.67 & 51.33 & 42 & 58 \\
\hline 16 & 500 & 15 & 5 & 14.35 & 85.65 & 13.33 & 86.67 \\
\hline 17 & 500 & 15 & 10 & 33.41 & 66.59 & 24 & 76 \\
\hline 18 & 500 & 15 & 15 & 42.12 & 57.88 & 33.33 & 66.67 \\
\hline 19 & 750 & 5 & 5 & 23.88 & 76.12 & 21.62 & 78.38 \\
\hline 20 & 750 & 5 & 10 & 47.33 & 52.67 & 43.24 & 56.76 \\
\hline 21 & 750 & 5 & 15 & 63.48 & 36.52 & 51.35 & 48.65 \\
\hline 22 & 750 & 10 & 5 & 26.37 & 73.63 & 28 & 72 \\
\hline 23 & 750 & 10 & 10 & 46.07 & 53.93 & 38.67 & 61.33 \\
\hline 24 & 750 & 10 & 15 & 64 & 36 & 58.67 & 41.33 \\
\hline 25 & 750 & 15 & 5 & 26.37 & 73.63 & 18.75 & 81.25 \\
\hline 26 & 750 & 15 & 10 & 49.92 & 50.08 & 46.43 & 53.57 \\
\hline 27 & 750 & 15 & 15 & 61.07 & 38.93 & 45.54 & 54.46 \\
\hline
\end{tabular}

- The aversion is not only experienced by disbelievers but also believers.

A visual representation of simulation of case 14 is given in Figure. 3 (d). Next, we analyze three interesting cases, resulting in norm aversion qualitatively. It has been observed that case $21 \& 24$ display aversion of unpopular norm whereas we can see partial aversion in case 27 .

In case 21 , we observe that the unpopular norm is averted at approximately 7.37 ticks. The graph in Figure. 4 shows that the disbelievers enforcing alternative norm (other than the unpopular norm), increasing afterwards, averting the unpopular norm. In addition to this, we also observe that the number of true believers enforcing the unpopular norm gradually decline up to tick 9.55, and start averting the norm afterwards.

In case 24 , we observe that the unpopular norm is averted more quickly, at 6.75 ticks. The graph in Figure. 5 shows that the disbelievers enforcing alternative norm (other than the unpopular norm) increase afterwards, averting the unpopular norm. In addition to this we also observe that the number of true believers enforcing the unpopular norm gradually decline up to tick 6 , and start averting the norm afterwards.

In case 27 , we observe that the unpopular norm is averted more quickly, at 6.74 ticks. The graph in Figure. 6 shows that the disbelievers enforcing alternative norm (other than the unpopular norm) increase afterwards, averting the unpopular norm. Contrary to this, we observe that the number of true believers enforcing the unpopular norm gradually decline but does not fall below the percentage of false believers thereby indicating that unpopular norm is only partially averted as shown in Figure. 6.

\section{CONCLUSiON}

In this paper, an agent-based simulation model of unpopular norm aversion is presented. We have modeled a theorydriven computational specifications of Emperor's Dilemma into an agent-based simulation, to understand the conditions that result in emergence of unpopular norms. The reciprocal nature of persistence and aversion of norms is utilized to define situations under which these norms can be changed and averted. Following is concluded from the analyzes of the simulation results. The percentage of agents averting the unpopular norm increases with increase in population. Further, the percentage of agents averting the unpopular norm increases with increase in agents actively participating in averting the unpopular norm.

\section{REFERENCES}

[1] H. P. Young et al., Social norms. Department of Economics, University of Oxford, 2007.

[2] D. Kübler, "On the regulation of social norms," Journal of Law, Economics, and Organization, vol. 17, no. 2, pp. 449-476, 2001.

[3] C. F. Blake, "Foot-binding in neo-confucian china and the appropriation of female labor," Signs, vol. 19, no. 3, pp. 676-712, 1994.

[4] B. Essén and S. Johnsdotter, "Female genital mutilation in the west: traditional circumcision versus genital cosmetic surgery," Acta Obstetricia et Gynecologica Scandinavica, vol. 83, no. 7, pp. 611-613, 2004.

[5] M. M. Maaga, Hearing the voices of Jonestown. Syracuse University Press, 1998.

[6] C. Bicchieri and Y. Fukui, "The great illusion: Ignorance, informational cascades, and the persistence of unpopular norms," Business Ethics Quarterly, vol. 9, no. 01, pp. 127-155, 1999.

[7] D. Easley and J. Kleinberg, Networks, crowds, and markets: Reasoning about a highly connected world. Cambridge University Press, 2010.

[8] M. Macy and A. Flache, "Social dynamics from the bottom up: Agentbased models of social interaction," The Oxford handbook of analytical sociology, pp. 245-268, 2009.

[9] D. Centola, J. C. Gonzalez-Avella, V. M. Eguiluz, and M. San Miguel, "Homophily, cultural drift, and the co-evolution of cultural groups," Journal of Conflict Resolution, vol. 51, no. 6, pp. 905-929, 2007.

[10] D. Centola and M. Macy, "Complex contagions and the weakness of long ties1," American journal of Sociology, vol. 113, no. 3, pp. 702734, 2007.

[11] M. Michaeli and D. Spiro, "Inverted preferences and skewed norm," Journal of Philosophical Logic, 2013. 

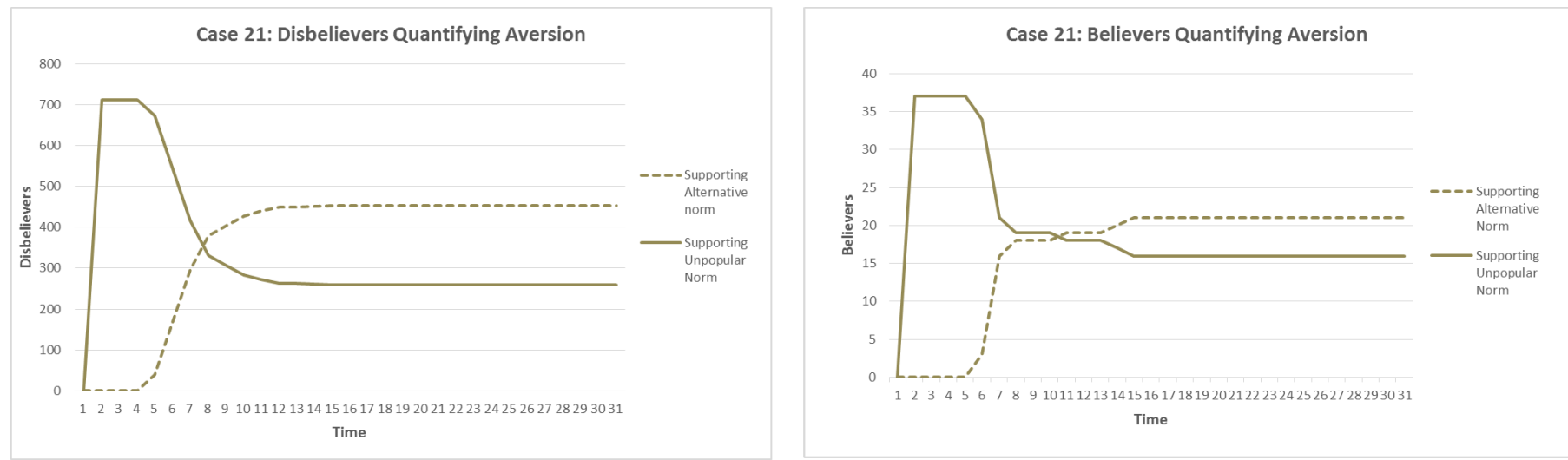

Fig. 4: Time-line of Case 21.
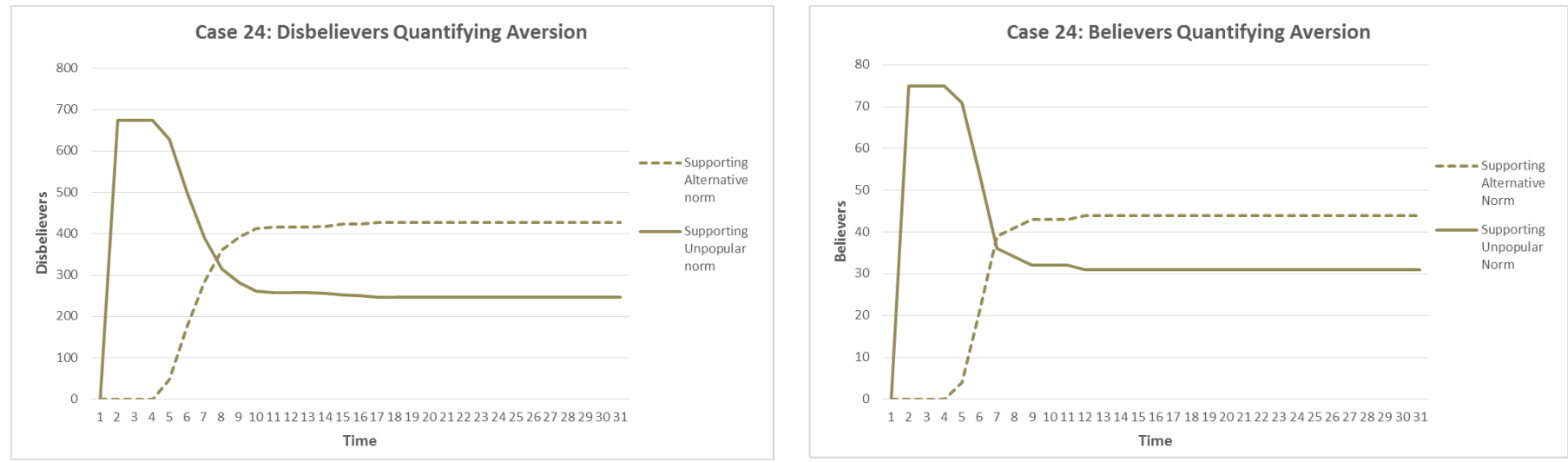

Fig. 5: Time-line of Case 24.
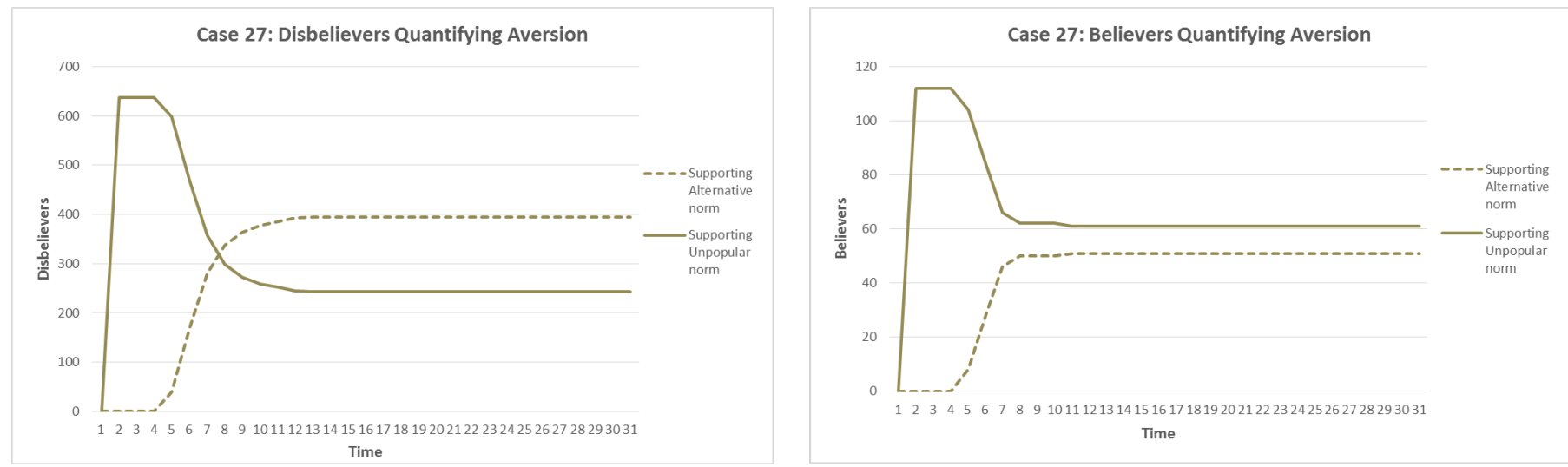

Fig. 6: Time-line of Case 27.

[12] M. Granovetter, "Threshold models of collective behavior," The American Journal of Sociology, vol. 83, no. 6, pp. 1420-1443, 1978.

[13] A. C. Martins, "Trust in the coda model: Opinion dynamics and the reliability of other agents," Physics Letters A, vol. 377, no. 37, p. 23332339, 2013.

[14] L. Muchnik, S. Aral, and S. J. Taylor, "Social influence bias: A randomized experiment," Science, vol. 341, no. 6146, pp. 647-651, 2013.

[15] M. A. Pachucki and R. L. Breiger, "Cultural holes: Beyond relationality in social networks and culture," Annual review of sociology, vol. 36, pp.
205-224, 2010.

[16] D. Centola, R. Willer, and M. Macy, "The emperors dilemma: A computational model of self-enforcing norms1," American Journal of Sociology, vol. 110, no. 4, pp. 1009-1040, 2005.

[17] D. Helbing, W. Yu, K.-D. Opp, and H. Rauhut, "Conditions for the emergence of shared norms in populations with incompatible preferences," Plos One, vol. 9, no. 8, pp. 1-14, 2014.

[18] P. G. Hansen, V. F. Hendricks, and R. K. Rendsvig, "Infostorms," Metaphilosophy, vol. 44, no. 3, pp. 301-326, 2013.

[19] D. Mungovan, E. Howley, and J. Duggan, "The influence of random in- 
teractions and decision heuristics on norm evolution in social networks," Computational and Mathematical Organization Theory, vol. 17, no. 2, pp. 152-178, 2011.

[20] J. U. Hansen, "A logic-based approach to pluralistic ignorance," in Logic and Interactive RAtionality Yearbook, vol. 2. The Institute for Logic, Lanuage and Computation, 2014, pp. 226-245.

[21] C. Proietti and E. J. Olsson, "A ddl approach to pluralistic ignorance and collective belief," Journal of Philosophical Logic, vol. 43, no. 2 , pp. 499-515, 2014

[22] R. Willer, K. Kuwabara, and M. W. Macy, "The false enforcement of unpopular norms," American Journal of Sociology, vol. 115, no. 2, pp. 451-490, 2009.

[23] K. Gerxhani and J. Bruggeman, "Time lag and communication in changing unpopular norms," Plos One, pp. 1-17, 2015.

[24] U. Wilensky, " $\{$ NetLogo $\}, " 1999$.

[25] A. Ferscha and K. Zia, "Lifebelt: Silent directional guidance for crowd evacuation," in Wearable Computers, 2009. ISWC'09. International Symposium on. IEEE, 2009, pp. 19-26. 